\title{
Analyzing bacterial community in pit mud of Yibin Baijiu in China using high throughput sequencing
}

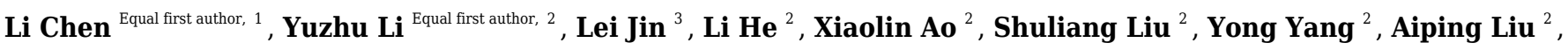 \\ Shujuan Chen ${ }^{\text {Corresp. } 2}{ }^{2}$, Likou Zou ${ }^{\text {Corresp. } 3}$ \\ ${ }^{1}$ Yibin Products Quality Supervision and Inspection Institute, Yibin, Sichuan, China \\ 2 College of Food Science, Sichuan Agricultural University, Ya'an, Sichuan, China \\ ${ }^{3}$ College of Resources, Sichuan Agricultural University, Chengdu, Sichuan, China \\ Corresponding Authors: Shujuan Chen, Likou Zou \\ Email address: chenshujuan1@163.com, zoulikou@sicau.edu.cn
}

"Yibin Baijiu" (YB) is a special Chinese strong-aroma Baijiu (CSAB) that originated in Yibin, a city in western China. YB is fermented in cellars lined with pit mud (PM), the microbiota in which may affect YB quality. In this study, high throughput sequencing of the 16S rRNA gene was used to demonstrate the bacterial community structure and diversity in PM of YB. In addition, the physicochemical characteristics of PM were also analyzed, including moisture content, $\mathrm{pH}$, and available phosphorous, ammonia nitrogen, and humic acid levels. Results showed that Firmicutes was the dominant phylum in all PM samples with abundance $>70.0 \%$, followed by Euryarchaeota (11.3\%), Bacteroidetes (6.5\%), Synergistetes (3.0\%), Actinobacteria (1.4\%), and Proteobacteria (1.2\%). Furthermore, 14 different genera with average relative abundance of $>1 \%$ were detected. The Chaol and Shannon indexes did not vary significantly between the sub-layer and middle-layer PM $(P>$ 0.05). However, Linear discriminant analysis Effect Size (LEfSe) analysis showed that the relative abundance of Lactobacillus in the sub-layer PM was significantly higher than in middle-layer PM. pH differed significantly $(P<0.05)$ between the two groups. Canonical correspondence analysis revealed that bacterial community in PM correlated significantly with available phosphorous content and $\mathrm{pH}$. Our study provides basic data for further elucidating the diversity of microbiota in the PM of YB and the potential mechanism of Baijiu production. 


\section{Analyzing bacterial community in pit mud of Yibin 2 Baijiu in China using high throughput sequencing}

3

4

5

6

7

8

9

10 11

Li Chen a, 1, Yuzhu Li ${ }^{b}, 1$, Lei Jin ${ }^{c}$, Li He $^{b}$, Xiaolin Ao ${ }^{b}$, Shuliang Liu ${ }^{b}$, Yong Yang ${ }^{b}$, Aiping Liu ${ }^{b}$, Shujuan Chen ${ }^{\text {,* }}$, Likou Zou ${ }^{\text {c,* }}$

a Yibin Products Quality Supervision and Inspection Institute, Yibin, Sichuan, China

${ }^{\mathrm{b}}$ College of Food Science, Sichuan Agricultural University, Ya'an, Sichuan, China

${ }^{c}$ College of Resources, Sichuan Agricultural University, Chengdu, Sichuan, China

Corresponding Author:

Shujuan Chen

No.46 Xinkang Road, Yucheng District, Ya'an City, Sichuan Province, P.R. 625014, China

Email address: chenshujuan1@163.com

Likou Zou

No.211 Huimin Road, Wenjiang District, Chengdu City, Sichuan Province, P.R. 611130, China

Email address: zoulikou@sicau.edu.cn

${ }^{1}$ The authors contributed equally to this work 


\section{Abstract}

23

24

25

26

27

28

29

30

31

32

33

34

35

36

37

38

39

40

41

42

43

44

45

46

47

48

49

50

51

52

53

54

55

56

57

58

59

"Yibin Baijiu" (YB) is a special Chinese strong-aroma Baijiu (CSAB) that originated in Yibin, a city in western China. YB is fermented in cellars lined with pit mud (PM), the microbiota in which may affect YB quality. In this study, high throughput sequencing of the 16S rRNA gene was used to demonstrate the bacterial community structure and diversity in PM of YB. In addition, the physicochemical characteristics of PM were also analyzed, including moisture content, $\mathrm{pH}$, and available phosphorous, ammonia nitrogen, and humic acid levels. Results showed that Firmicutes was the dominant phylum in all PM samples with abundance $>70.0 \%$, followed by Euryarchaeota (11.3\%), Bacteroidetes (6.5\%), Synergistetes (3.0\%), Actinobacteria $(1.4 \%)$, and Proteobacteria (1.2\%). Furthermore, 14 different genera with average relative abundance of $>1 \%$ were detected. The Chao 1 and Shannon indexes did not vary significantly between the sub-layer and middle-layer PM $(P>0.05)$. However, Linear discriminant analysis Effect Size (LEfSe) analysis showed that the relative abundance of Lactobacillus in the sub-layer PM was significantly higher than in middle-layer PM. pH differed significantly $(P<0.05)$ between the two groups. Canonical correspondence analysis revealed that bacterial community in $\mathrm{PM}$ correlated significantly with available phosphorous content and $\mathrm{pH}$. Our study provides basic data for further elucidating the diversity of microbiota in the PM of YB and the potential mechanism of Baijiu production.

\section{Introduction}

Baijiu is one of the six most famous distilled liquors worldwide (Fan \& Qian, 2006; Jin, Zhu, \& Xu, 2017; McGovern et al., 2004). It includes four main aroma types: strong, light, sauce, and rice. Among these, the Chinese strong-aroma Baijiu (CSAB) accounts for $70 \%$ of the total Baijiu production (Liu \& Sun, 2018; Liu, Tang, Zhao, et al., 2017). Yibin is one of the birth places of CSAB culture (Zou, Zhao, \& Luo, 2018; You et al., 2016), and it is located at the confluence of the Min River, Jinsha River, and Yangtze River, which has good water quality. In addition, it has unique weak acid clay soil with strong viscosity, good water retention property, and abundant minerals, which makes it an excellent natural material for making cellars (Tang et al., 2012). Furthermore, Yibin enjoys a mid-subtropical humid monsoon climate, which is perfect for the growth of liquor-making microbes (Zhao et al., 2013). Owing to these advantages, Yibin is wellsuited for the development of uniquely flavored "Yibin Baijiu" (YB). It is noteworthy that YB has been successfully declared the national geographical indication product of 2010 (Yang et al., 2017).

YB is produced by distilling mixed and fermented grains such as sorghum, glutinous rice, rice, wheat, and corn, and is characterized by sweetness, strong aroma, and lasting aftertaste (Zheng et al., 2013; Zheng \& Han, 2016). YB is produced via a traditional solid-state fermentation process that occurs in fermentation pits (approximate dimensions: length, 2.0-3.5 m; width, 2.0-3.0 m; depth, 2.3-2.5 m) (Barrios-González, 2012), the inner walls of which are covered with a thick layer of pit mud (PM). The PM is a complex ecosystem consisting of a variety of microbial 
60 communities, such as bacteria, archaea, and fungi (Liu et al., 2017). Bacterial communities in

61 PM can break down macromolecules into small peptides and monosaccharides, which produce 62 aromatic compounds responsible for the product flavor (Tao et al., 2014; Zhao, Zheng, Zhou, \&

63 Shi, 2012). However, the underlying mechanism remains to be identified. To understand the role

64 of the microorganisms in YB fermentation and optimize the brewing technology, the

65 composition and diversity of the microbial population in PM has to be investigated.

66 Since the 1960s, researchers have used traditional culture methods for identifying the

67 microbiota in PM (Wu, Qi, Xue, Guo, \& Jie, 1980). Clostridium, Bacillus, Pseudomonas, and

68 Sporolactobacillus were isolated and identified using traditional microbial classification and

69 identification methods (Yue, Hang, Liu, Hu, \& Zheng, 2007). However, these methods detected

$70<1 \%$ of culturable microorganisms, which failed to reveal the complete structural characteristics

71 of the PM microbial ecosystem (Amann, Ludwig, \& Schleifer, 1995). Subsequently, molecular

72 methods, such as polymerase chain reaction-denaturing gradient gel electrophoresis (PCR-

73 DGGE) (Deng et al., 2012; Huang, Xiong, Hu, Liang, \& Zhao, 2017), phospholipid fatty acid

74 (PLFA) analysis (Zheng et al., 2013), and clone library analysis of the 16S rRNA gene (Ye et al.,

75 2013), have been used extensively. These methods not only circumvented the tedious process of

76 microbial cultivation, but also contributed to our understanding of the PM ecosystem. Thus,

77 Firmicutes, Proteobacteria, Bacteroidetes, Actinobacteria, and Synergistetes were found to be

78 predominant in many PM bacterial communities (Ding, Wu, Huang, Li, \& Zhou, 2014; Liang,

79 Luo, Zhang, Wu, \& Zhang, 2016). Although these molecular methods provided the first

80 description of the diverse microbial populations in PM, the extent of bacterial diversity remains

81 unexplored. Currently, 16S rRNA gene high throughput sequencing is being used for microbial

82 identification (Liu, 2017; Zheng et al., 2015). However, studies investigating the bacterial

83 community structure and diversity in PM of YB using high-throughput sequencing strategies are

84 lacking. Furthermore, Yibin has a brewing history of nearly a thousand years, suitable ecological

85 factors, and relatively special brewing techniques, which have gradually contributed to the

86 formation of a unique and stable bacterial community structure in PM. Therefore, considering

87 the complexity of PM microorganisms, it is necessary to study the PM of YB to obtain more

88 information regarding PM bacterial communities.

89 Hence, this study aimed to investigate the bacterial community structure and diversity in PM

90 of YB using high-throughput sequencing. Furthermore, the physicochemical characteristics of

91 PM were determined to investigate the correlation between bacterial community structure and

92 environmental factors, which will assist us in accumulating basic data for further elucidating the

93 mechanism of Baijiu brewing, and will promote the long-term development of YB.

\section{Materials \& Methods}

\section{Sampling}

96 PM samples were collected in December 2017 from 13 distilleries located in Yibin $\left(28^{\circ} 76^{\prime} \mathrm{N}\right.$,

$\left.97104^{\circ} 63^{\prime} \mathrm{E}\right)$. In total, 68 samples were collected, among which 37 were collected from the middle

98 layer of cellar walls and 31 were collected from the substrate layer (100 g PM at each position). 
99 The PM sampling sites are shown in Figure S1. PM samples were numbered according to the

100

101

102

103

104

105

106

107

108

109

110

111

112

113

114

115

116

117

118

119

120

121

122

123

124

125

126

127

128

129

130

131

132

133

134

135

136

137

138 information regarding the distillery, cellar, and layer, and were divided into two groups, the middle-layer and the sub-layer (Table S1). The samples were aseptically collected in sterilized bags and maintained at low temperature during transport from the distilleries to the laboratory. Physicochemical properties

The basic physicochemical properties of PM, including moisture content, $\mathrm{pH}$, and available phosphorous, ammonia nitrogen, and humic acid content were also determined as described previously (Shen, 2014).

DNA extraction, polymerase chain reaction (PCR), and Illumina HiSeq sequencing DNA was extracted from the PM using the FastDNA SPIN kit for soil (Mpbio, USA) according to the manufacturer's instructions. The crude DNA was quantified based on the absorbance at $260 \mathrm{~nm}$ using a NanoDrop ${ }^{\mathrm{TM}}$ one spectrophotometer (Thermo Fisher Scientific, USA), and the purity was assessed using agarose gel electrophoresis (Beijing Liuyi Biological Technology Co., Ltd. China).

To amplify the V3-V4 regions of $16 \mathrm{~S}$ rRNA genes, a pair of universal bacterial primers was used in PCR: a forward primer (341F, CCTAYGGGRBGCASCAG) and a reverse primer (806R, GGACTACHVGGGTWTCTAAT). The reaction mixtures $(25 \mu \mathrm{l})$ contained $5 \mu \mathrm{l}$ of $5 \times$

TransStart Fastpfu buffer (Transgen Biotech), 50 ng DNA sample, $1 \mu$ of each primer, $2 \mu 12.5$ mM dNTPs, and $0.5 \mu \mathrm{l}$ TransStart Fastpfu DNA polymerase (Transgen Biotech). The PCR conditions were as follows: $2 \mathrm{~min}$ at $95^{\circ} \mathrm{C} ; 30$ cycles of $20 \mathrm{~s}$ at $95^{\circ} \mathrm{C}, 30 \mathrm{~s}$ at $55^{\circ} \mathrm{C}, 30 \mathrm{~s}$ at $72^{\circ} \mathrm{C}$; final extension at $72{ }^{\circ} \mathrm{C}$ for $5 \mathrm{~min}$ and cooling at $4^{\circ} \mathrm{C}$. The PCR products were purified using a universal PCR purification kit (Tiangen, Beijin, China) and sent to a commercial sequencing company for high-throughput sequencing using the Illumina Hiseq sequencing platform (MeiYin Health Technology (Beijing) Co., Ltd., China).

\section{Nucleotide sequence accession numbers}

The HiSeq sequencing data were submitted to the Sequence Read Archive (SRA) of the NCBI database as BioProject ID PRJNA597727 (http://www.ncbi.nlm.nih.gov/).

Data analysis

All the raw HiSeq-generated 16S rRNA gene sequencing data were processed using the QIIME pipeline (V1.9.1). After initial quality control (QC) processing, pairs of reads were merged using FLASH (V1.2.7, Magoč \& Salzberg, 2011) to acquire raw tags. Then, they were screened using the QIIME function (Caporaso et al., 2010) to obtain clean tags. Chimeras were detected and removed using the Uchime algorithm to obtain effective tags (Edgar, Haas, Clemente, Quince, \& Knight, 2011). The effective tags, at a similarity threshold of 97\%, were grouped into the same operational taxonomic units (OTUs). A representative sequence for each OTU was extracted and annotated using the SILVA database V119 (Quast et al., 2012).

Community richness and diversity were estimated using the Chaol (Chao \& Bunge, 2002) and Shannon indices (Shannon, 1997), respectively. Bacterial community differences in two groups of PM were evaluated using principal coordinates analysis (PCoA) in Fast UniFrac (Hamady, Lozupone, \& Knight, 2010). Linear discriminant analysis effect size (LEfSe) was used to

Peer] reviewing PDF | (2019:12:44183:1:0:NEW 31 Mar 2020) 
139 compare the significance of species-specific differences between the two groups (Segata et al.,

140 2011). Canonical correspondence analysis (CCA) between bacterial communities in the PM and

141 physicochemical properties was performed using the vegan package in $\mathrm{R}$ (Oksanen et al., 2018).

142 All the statistical analyzes, including Spearman's test, were performed with the SPSS software

143 (V22.0, IBM Corp., Armonk, NY, USA). All tests for significance were two-sided, and $P$-values $144<0.05$ were considered statistically significant.

\section{Results}

146 Physicochemical properties of the PM

147 The physicochemical characteristics of the PM of the two groups are shown in Table S2. Among 148 the physicochemical indicators analyzed using one-way analysis of variance (ANOVA) of SPSS

149 Statistics $22, \mathrm{pH}$ differed significantly $(P<0.05)$ between the two groups. However, no

150 significant differences in moisture content, and ammonia nitrogen, available phosphorus, and

151 humic acid levels were observed.

152 Diversity of bacterial community in PM

153 In total, 68 samples were analyzed for amplicon sequencing. On an average, 99,639 clean tags

154

155

156

157

158

159

160

161

162

163

164 were generated for each sample with a minimum of 53,888 tags. In total, 53,493-208,410 effective tags for each sample with an average sequence length of $444 \mathrm{bp}$ were generated. Rarefaction curves were used to assess the degree of completion of a taxonomic survey (Good, 1953). The rarefaction curve showed that all curves approached the saturation plateau, proving that the identified sequences of PM samples represented almost all the bacterial sequences (Figure S1).

Eventually, 220,790 operational taxonomic units (OTUs) were detected based on 97\% similarity in 16S rRNA gene sequences, of which the middle-layer group and sub-layer group had 186,289 and 148,904 OTUs, respectively. Furthermore, 114,403 OTUs were shared between the two groups. The mutual and unique OTUs for the two groups are shown in Venn diagrams (Figure S2).

The bacterial richness (Chao1) indices for all samples ranged from 1,214.30 to 42,441.82, and were fairly similar in the two different groups, with $11,296.93 \pm 4,816.73$ in the middle-layer group and 10,549.07 \pm 7,638.48 in the sub-layer group. Furthermore, the diversity (Shannon)

169 indices were $7.82 \pm 1.68$ in the middle-layer group and $7.57 \pm 1.21$ in the sub-layer group. The Chao1 and Shannon indexes did not vary significantly between the two groups $(P>0.05)$, suggesting similar overall species diversity (Figure 1). Furthermore, the PCoA analysis showed that there was no explicit clustering of samples within the groups, demonstrating significant inter-individual variations in the bacterial communities (Figure 2).

173 Bacterial community structure in PM In total, 49 phyla, 332 families, and 811 genera were identified in all the samples (Table 1). The most abundant phylum was Firmicutes (71.9\%), followed by Euryarchaeota (11.3\%),

176 Bacteroidetes (6.5\%), Synergistetes (3.0\%), Actinobacteria (1.4\%), Proteobacteria (1.2\%),

177 Cloacimonetes $(0.6 \%)$, Tenericutes $(0.4 \%)$, Chloroflexi (0.3\%), and Cyanobacteria $(0.2 \%)$ 
178 (Figure 3). Most notably, Firmicutes constituted more than $70.0 \%$ of the bacterial population in 179 all the groups; in addition, Euryarchaeota and Bacteroidetes were also predominant in all groups 180 with average relative abundances $>5 \%$. The proportions of these three major phyla in the 181 middle-layer and sub-layer group were $71.2 \%$ and $72.3 \%, 12.7 \%$ and $10.0 \%$, and $6.9 \%$ and $1826.3 \%$, respectively.

183 At the genus level, dominate genera were similar between the two groups. Fourteen genera 184 dominated all the samples (average relative abundance $>1 \%$ in all groups), including 185 Ruminiclostridium 5 (17.9\%), Gelria (8.4\%), Methanoculleus (4.9\%), Syntrophomonas (3.8\%), 186 Lactobacillus (3.6\%), Syntrophaceticus (2.8\%), Proteiniphilum (2.7\%), Aminobacterium (2.7\%), 187 Petrimonas (2.4\%), Sedimentibacter (2.1\%), Methanosarcina (1.9\%), Methanobacterium 188 (1.8\%), Methanobrevibacter (1.8\%), and Caldicoprobacter (1.7\%). The most abundant 10 genera are shown in Figure 4. In addition, the relative abundance of Clostridium sensu stricto 14 and Clostridium sensu stricto 12 also exceeded 1\% in the sub-layer group, being $1.8 \%$ and 1.7\%, respectively. LEfSe was used to determine the classified bacterial taxa with significant abundance differences between two groups. The results of the LEfSe analysis showed that Lactobacillus is the key discrepant bacteria, and that the relative abundance of Lactobacillus in the sub-layer PM was significantly higher than that in middle-layer PM (Figure 5). Comparison of dominant bacterial communities in different Baijiu PM Based on the results of previous studies, we compared the dominant microbiota of strong-aroma Baijiu in different regions to ascertain the common dominant bacteria, and the unique dominant bacteria of YB (Table 2). Strong-aroma Baijiu PM contained four mutually dominant bacteria in five different regions (Mianzhu, Luzhou, Jiangsu, Hunan, Yibin), namely, Lactobacillus,

200 Sedimentibacter, Syntrophomonas, and Methanobrevibacter. In addition, it is noteworthy that Petrimonas, Clostridium, Methanosarcina, Methanoculleus, and Methanobacterium were also

203

204 detected in our study with the relative abundance above $1 \%$, and almost all of them belonged to phylum Euryarchaeota. Three unique bacteria were also detected in YB PM, namely Ruminiclostridium 5, Gelria, and Syntrophaceticus.

205

206

207

In addition, the common dominant microorganisms of strong-aroma Baijiu and those of other aroma types (miscellaneous-aroma and sauce-aroma) were compared. The dominant microbiota

208

209 composition in the Baijiu PM of different aroma types differed considerably. Only Lactobacillus was the dominant microorganism shared by three aroma types of Baijiu PM. In addition,

210 Enterobacter was the other dominant microorganism shared by miscellaneous-aroma and saucearoma, which was not found in strong-aroma Baijiu PM. The detailed information regarding the

211 dominant microflora of the three aroma types of Baijiu PM is shown in Table 3.

\section{Correlation between environmental factors and bacterial community}

213 CCA was performed to identify the major environmental factors that affected the variation in 214 bacterial communities. The contribution of environmental factors was as follows: available 215 phosphorous $\left(\mathrm{R}^{2}=0.237, P=0.0005\right)>\mathrm{pH}\left(\mathrm{R}^{2}=0.210, P=0.0015\right)>$ humic acid $\left(\mathrm{R}^{2}=0.056, P\right.$ $216=0.1559)>$ ammonia nitrogen $\left(\mathrm{R}^{2}=0.053, P=0.1724\right)>$ moisture content $\left(\mathrm{R}^{2}=0.049, P=\right.$ 217 0.1959). The results of CCA indicated that among these five factors, available phosphorous and 
$218 \mathrm{pH}$ maximally affected PM bacterial community structure. Furthermore, the Spearman's

219 correlation coefficient was determined between environmental factors and the most abundant 35

220 genera were performed. The results showed that $\mathrm{pH}$ correlated negatively with the relative

221 abundance of Clostridium sensu stricto $12(\mathrm{R}=-0.502, P=0.002)$, Lachnoclostridium $(\mathrm{R}=$

$222-0.473, P=0.008)$, and Lactobacillus $(\mathrm{R}=-0.451, P=0.020)$ at the genus level (Figure 6).

223 Discussion

224 This is the first study to use the Illumina Hiseq sequencing platform for investigating the

225

226

227

228

229

230

231

232

233

234

235

236

237

238

239

240

241

242

243

244

245

246

247

248

249

250

251

252

253

254

255

256 population diversity of bacteria in the middle-layer and sub-layer PM of YB. In addition, the relationship between bacterial community structure and environmental factors was also assessed. The results indicated that the dominant bacteria in PM of YB were different from that of other regions and aroma types of Baijiu. The $\mathrm{pH}$ and available phosphorus content significantly affected the PM bacterial community structure.

According to previous studies, the total number of effective tags of the 16S rRNA gene of microorganisms in PM obtained from the Roche 454 or Illumina Miseq sequencing platforms were less than 10,000 (Liu et al., 2017; Tao et al., 2014). In our study, more than 200,000 effective tags were detected in a single sample with an average of 94,023 tags for all samples. Thus, the result of sequencing can adequately reflect most of the sample diversity noted.

In total, 49 phyla, 332 families and 811 genera were detected, which may be helpful for further understanding the phylogenetic status of numerous uncultured microbes in the PM ecosystem. Although the relative abundance of Firmicutes, Euryarchaeota, Bacteroidetes, and Synergistetes differed slightly between middle-layer and sub-layer samples, they were still the key components of the microbiota in the PM of YB. Previous studies also showed that Firmicutes, Euryarchaeota and Bacteroidetes were the three most abundant phyla in PM (Liang et al., 2015; Liu et al., 2017; Tao et al., 2014). Firmicutes include syntropyhic bacteria that can degrade volatile fatty acids such as butyrate and its analogs, and is represented mainly by the Clostridia and Bacilli (Garcia-Peña et al., 2011). Euryarchaeota includes diverse groups of methanogens that are interspersed with non-methanogenic lineages. Methanogens are often symbiotic with hexanoic acid bacteria and can effectively increase the content of ethyl hexanoate in strong-aroma Baijiu via "hydrogen transfer" effect between species (Zhao et al., 2017). Bacteroidetes includes three major types of bacteria: Bacteroidia, Flavobacteriia, and Sphingobacteriia. Most of them are capable of degrading macromolecular carbohydrates such as cellulose to produce acid (Guo, Cheng, Sun, Zhu \& Wu, 2014). All these phyla in PM played a vital role in Baijiu fermentation.

At the genus level, the dominant bacteria in PM from different regions producing strongaroma Baijiu were compared. In total, 11 dominant genera were detected by Liu et al. (2017) in CSAB's PM collected from Luzhou, Sichuan province (about 110 kilometers from Yibin city), among which 8 genera were also the dominant bacteria in YB PM. Tao et al. (2014) reported 14 dominant genera in CSAB's PM collected from Mianzhu, Sichuan province (about 300 kilometers from Yibin city), 6 of which were different from those of YB PM. In comparison, we 
257

258

259

260

261

262

263

264

265

266

267

268

269

270

271

272

273

274

275

276

277

278

279

280

281

282

283

284

285

286

287

288

289

290

291

292

293

294

295

296

observed that the composition of the dominant genera in PM in the Mianzhu area differed from that in the Yibin area due to their geographical distance. In contrast 7 of the 17 dominant genera in Jiangsu area (about 1,700 kilometers away from Yibin city) Baijiu PM were shared by YB PM. The dominant genera in Jiangsu area Baijiu PM and YB PM differed significantly, which further suggested that geographical location considerably affects Baijiu PM bacterial community. Previous studies have demonstrated that the composition of PM bacterial community varied by geographical location (Huang, Wang, Wei, Liu, \& Li, 2015; Liang et al., 2016). Interestingly, the dominant genera in the Baijiu PM of Hunan region, which is 470 kilometers away from Yibin City, was also similar to the dominant genera in YB PM. It was speculated that the identical latitude of Hunan and Yibin, which resulted in both having the same climate, especially temperature, significantly affected the diversity and structure of the microbiota. However, the farther are two places, the more is the similarity in the dominant genera of the Baijiu PMs from these areas.

The dominant genera in the PM of strong aroma Baijiu differed considerably from those of Baijius of other aromas (Table 3). Obviously, the differences in these dominant bacteria contributed significantly to the formation of different aroma types of Baijiu. Lactobacillus was a dominant genus among Baijiu PMs of several aroma types, (Huang, Xiong, Hu, Liang, \& Zhao, 2017; Wang, Zhang, \& Liu, 2016; Hu, Du, Ren, \& Xu, 2016), leading to the speculation that Lactobacillus was critical for Baijiu brewing. Gelria was found to be the dominant genus in our study, which has been rarely reported in previous studies. In syntrophic association with a hydrogenotrophic methanogen, Gelria can utilize various amino acids and sugars to produce acetate, propionates, $\mathrm{H}_{2}, \mathrm{NH}_{4}{ }^{+}$, and $\mathrm{CO}_{2}$ (Plugge et al., 2002). Fan et al. (2019) reported that Gelria showed a significant positive correlation with the content of major flavor substances such as alcohols, esters, and aldehydes in Baijiu. Therefore, as a unique dominant genus of YB PM, we speculated that Gelria may contribute to the formation of unique flavor of YB. Furthermore, a positive correlation between humic acid content and Gelria abundance was evident in the heat map (Figure 6). Humic acid is an important organic matter in PM, which is formed by the accumulation of microbial metabolites and can completely reflect the ability of PM to supply nutrients to microorganisms (Zhu et al., 2018). We speculated that the metabolic activity of Gelria promoted the formation of humic acid, which in turn provided nutrition for the growth and metabolism of Gelria.

The difference between the physicochemical indicators of the middle-layer and sub-layer PM of YB was analyzed using ANOVA. Results showed that the $\mathrm{pH}$ of the sub-layer was significantly lower than that of the middle-layer. Meanwhile, CCA analysis showed that the $\mathrm{pH}$ of PM correlated considerably with the bacterial community structure in PM. Spearman's analysis revealed that the Clostridium sensu stricto 12, Lachnoclostridium, and Lactobacillus were correlated negatively with $\mathrm{pH}$ value. LEfSe also showed that Lactobacillus and Clostridiaceae_1 were significantly less abundant in the middle-layer of PM with higher pH. In particular, the above three genera that were significantly related to $\mathrm{PM} \mathrm{pH}$ belonged to Firmicutes, the most abundant phylum. Among them, Clostridium sensu stricto 12 and 
297

298

299

300

301

302

303

304

305

306

307

308

309

310

311

312

313

314

315

316

317

318

319

320

321

322

323

324

325

326

327

328

329

330

331

332

333

334

Lachnoclostridium belonged to the order Clostridiales, while Lactobacillus belonged to the order Lactobacillales. Bacteria of order Clostridiales possess the metabolic ability to convert organic substances into organic acids (such as butyric, caproic acids, etc.), alcohols, $\mathrm{CO}_{2} / \mathrm{H}_{2}$, and minerals (Kenealy, Cao, \& Weimer, 1995; Zheng et al., 2013). Furthermore, Clostridiales contributed to the formation of ethyl butyrate and ethyl caproate when butyric and caproic acids reacted with alcohols via enzymatic and non-enzymatic catalysis (Ding, Wu, Zhang, Zheng, \& Zhou, 2014). Lactobacillales also plays an important role in Baijiu brewing, as the lactic acid produced can form ethyl lactate. Ethyl lactate, ethyl butyrate, and ethyl caproate are vital flavor compounds in CSAB (Fan \& Qian, 2005).

Interestingly, a strong correlation of the bacterial community with the available phosphorous level was also observed using CCA analysis. The available phosphorus provides nutrients for the growth and reproduction of microorganisms. Phosphorus is present in the cell nuclear membrane and is a source of the energy substance ATP. Zheng et al. (2013) reported that the low pH and high content of available phosphorus in PM may have resulted in the orthogenesis of microorganisms and Clostridiales-, Lactobacillaceae-, and Bacillales-dominated microbial community structure. However, Spearman's analysis showed the absence of any significant correlation between bacterial presence and the available phosphorous level. This may be because Spearman's analysis only performed a pairwise correlation between environmental factors and the most abundant 35 genera and did not reflect the overall situation. In addition, the available phosphorous level may correlate significantly with some low-abundance genera in the PM, which was not evident in the Spearman's heat map.

In this study, we analyzed the community characteristics of bacteria in PM of YB, and observed that fungi also played an indispensable role in fermentation. We will study the community structure and diversity of fungi in the future. Furthermore, using a combination of metagenomic technology and metabolite detection technology, we will investigate the functional microorganisms that affect the quality and flavor of YB in PM. This will provide theoretical guidance and technical support for the application of functional bacteria in YB development.

\section{Conclusions}

This study demonstrated the population diversity of bacteria in the PM of YB from 13 distilleries using HiSeq sequencing. Our results suggested the presence of a complex community structure and abundant species in the PM of YB. No significant difference was observed in bacterial diversity between middle-layer and sub-layer PM. The LEfSe analysis showed that the relative abundance of Lactobacillus in the sub-layer PM was significantly higher than that in middle-layer PM. pH differed significantly $(P<0.05)$ between the two groups. Available phosphorous and $\mathrm{pH}$ strongly affected bacterial community structure in PM. Our study provided basic information for further elucidating the diversity of microbiota in the PM of YB and the potential mechanism of Baijiu production.

\section{Acknowledgements}


335 This study was supported by Yibin Science and Technology Bureau (2017CNY001-4).

336 Conflicts of Interest: The authors declare no conflict of interest.

337

338

339

340

341

342

343

344

345

346

347

348

349

350

351

352

353

354

355

356

357

358

359

360

361

362

363

364

365

366

367

368

369

370

371

372

\section{References}

Amann, R. I., Ludwig, W., \& Schleifer, K. H. 1995. Phylogenetic identification and in situ detection of individual microbial cells without cultivation. Microbiological Reviews, 59 (1), 143-169. https://doi.org/10.1016/S0882-4010(95)90076-4

Barrios-González, J. 2012. Solid-state fermentation: Physiology of solid medium, its molecular basis and applications. Process Biochemistry, 47 (2), 175-185. https://doi.org/10.1016/j.procbio.2011.11.016

Bian, M. H., Ye, G. B., Yang, X. D., Ni, B., \& Li, D. Y. 2012. Research on Archaea Community Structure in the Pit Mud of Maotai-flavor Liquor. Liquor Making Science \& Technology (8), 51-53. https://doi.org/10.13746/j.njkj.2012.08.033

Caporaso, J. G., Kuczynski, J., Stombaugh, J., Bittinger, K., Bushman, F. D., Costello, E. K., Fierer, N., Pena, A. G., Goodrich, J. K., Gordon, J. I., Huttley, G. A., Kelley, S. T., Knights, D., Koenig, J. E., Ley, R. E., Lozupone, C. A., McDonald, D., Muegge, B. D., Pirrung, M., Reeder, J., Sevinsky, J. R., Turnbaugh, P. J., Walters, W. A., Widmann, J., Yatsunenko, T., Zaneveld, J., \& Knight, R. 2010. QIIME allows analysis of high-throughput community sequencing data. Nature Methods, 7 (5), 335-336. https://doi.org/10.1038/nmeth.f.303

Chao, A., \& Bunge, J. 2002. Estimating the number of species in a stochastic abundance model. Biometrics, 58 (3), 531-539. https://doi.org/10.1111/j.0006-341X.2002.00531.X

Deng, B., Shen, C. H., Shan, X. H., Ao, Z. H., Zhao, J. S., Shen, X. J., \& Huang, Z. G. 2012. PCR-DGGE analysis on microbial communities in pit mud of cellars used for different periods of time. Journal of the Institute of Brewing, 118 (1), 120-126. https://doi.org/10.1002/jib.2

Ding, X. F., Wu, C. D., Huang, J., Li, H., \& Zhou, R. Q. 2014. Eubacterial and archaeal community characteristics in the man-made pit mud revealed by combined PCR-DGGE and FISH analyses. Food Research International, 62, 1047-1053. https://doi.org/10.1016/j.foodres.2014.05.045

Ding, X. F., Wu, C. D., Zhang, L. Q., Zheng, J., \& Zhou, R. Q. 2014. Characterization of eubacterial and archaeal community diversity in the pit mud of Chinese Luzhou - flavor liquor by nested PCR-DGGE. World Journal of Microbiology Biotechnology, 30 (2), 605. https://doi.org/10.1007/s11274-013-1472-4

Edgar, R. C., Haas, B. J., Clemente, J. C., Quince, C., \& Knight, R. 2011. UCHIME improves sensitivity and speed of chimera detection. Bioinformatics, 27 (16), 2194-2200. https://doi.org/10.1093/bioinformatics/btr381

Fan, G., Fu, Z., Sun, B., Zhang, Y., Wang, X., Xia, Y., Huang M., \& Li, X. 2019. Roles of aging in the production of light-flavored Daqu. Journal of bioscience and bioengineering, 127 (3), 309-317. https://doi.org/10.1016/j.jbiosc.2018.08.005

Peer] reviewing PDF | (2019:12:44183:1:0:NEW 31 Mar 2020) 
373

374

375

376

377

378

379

380

381

382

383

384

385

386

387

388

389

390

391

392

393

394

395

396

397

398

399

400

401

402

403

404

405

406

407

408

409

410

411

412

Fan, W., \& Qian, M. C. 2005. Headspace Solid Phase Microextraction and Gas Chromatography-Olfactometry Dilution Analysis of Young and Aged Chinese "Yanghe Daqu” Liquors. Journal of Agricultural and Food Chemistry, 53 (20), 7931-7938. https://doi.org/10.1021/jf051011k

Fan, W., \& Qian, M. C. 2006. Characterization of aroma compounds of chinese "Wuliangye" and "Jiannanchun" liquors by aroma extract dilution analysis. Journal of Agricultural and Food Chemistry, 54 (7), 2695-2704. https://doi.org/10.1021/jf052635t

Garcia-Peña, E. I., Parameswaran, P., Kang, D. W., Canul-Chan, M., and Krajmalnik-Brown, R. 2011. Anaerobic digestion and co-digestion processes of vegetable and fruit residues: Process and microbial ecology, Bioresour. Technol. 102, 9447-9455. https://doi.org/10.1016/j.biortech.2011.07.068

Good, I. J. 1953. The Population Frequency of Species and the Estimation of Population Parameters. Biometrika, 40 (3/4), 237-264. https://doi.org/10.1093/biomet/40.3-4.237

Guo, X. H., Cheng, W., Sun F. O., Zhu, W. J. \& Wu, W. X. 2014. A comparison of microbial characteristics between the thermophilic and mesophilic anaerobic digesters exposed to elevated food waste loadings, Bioresour Technol, 152: 420-428. https://doi.org/10.1016/j.biortech.2013.11.012

Hamady, M., Lozupone, C., \& Knight, R. 2010. Fast UniFrac: facilitating high-throughput phylogenetic analyses of microbial communities including analysis of pyrosequencing and PhyloChip data. The ISME journal, 4(1), 17-27. https://doi.org/10.1038/ismej.2009.97

$\mathrm{Hu}, \mathrm{X} ., \mathrm{Du}, \mathrm{H} ., \mathrm{Ren}, \mathrm{C} ., \mathrm{X}$ X, Y. 2016. Illuminating Anaerobic Microbial Community and Cooccurrence Patterns across a Quality Gradient in Chinese Liquor Fermentation Pit Muds. Applied and environmental microbiology, 82 (8), 2506-2515. https://doi.org/10.1128/AEM.03409-15

Huang, Y. N., Xiong, X. M., Hu, Y. L., Liang, Y. X., \& Zhao, S. M. 2017. Bacterial community and diversity in pit mud of Baiyunbian liquor analyzed by PCR-DGGE and high-throughput sequencing. Microbiology China, 44 (2), 376-383. https://doi.org/10.13344/j.microbiol.china.160080

Huang, Z. G., Wang, Y. L., Wei, C. H., Liu, Y. M., \& Li, M. 2015. Diversity of Bacteria Community Structure in Pit Mud from Different Regions and Analysis of Its Correlations with Physicochemical Factors. Liquor Making Science \& Technology (11), 18-25. https://doi.org/10.13746/j.njkj.2015097

Jin, G., Zhu, Y., \& Xu, Y. 2017. Mystery behind Chinese liquor fermentation. Trends in Food Science \& Technology, 63, 18-28. https://doi.org/10.1016/j.tifs.2017.02.016

Kenealy, W. R., Cao, Y., \& Weimer, P. J. 1995. Production of caproic acid by cocultures of ruminal cellulolytic bacteria and Clostridium kluyveri grown on cellulose and ethanol. Applied Microbiology Biotechnology, 44 (3-4), 507-513. https://doi.org/10.1007/BF00169952

Liang, H., Li, W., Luo, Q., Liu, C., Wu, Z., \& Zhang, W. 2015. Analysis of the bacterial community in aged and aging pit mud of Chinese Luzhou-flavour liquor by combined 
413

414

415

416

417

418

419

420

421

422

423

424

425

426

427

428

429

430

431

432

433

434

435

436

437

438

439

440

441

442

443

444

445

446

447

448

449

450

451

PCR-DGGE and quantitative PCR assay. Journal of the Science of Food and Agriculture, 95 (13), 2729-2735. https://doi.org/10.1002/jsfa.7013

Liang, H. P., Luo, Q. C., Zhang, A., Wu, Z. Y., \& Zhang, W. X. 2016. Comparison of bacterial community in matured and degenerated pit mud from Chinese Luzhou-flavour liquor distillery in different regions. Journal of the Institute of Brewing, 122 (1), 48-54. https://doi.org/10.1002/jib.296

Liu, H., \& Sun, B. 2018. Effect of Fermentation Processing on the Flavor of Baijiu. Journal of Agricultural and Food Chemistry, 66 (22), 5425-5432.

https://doi.org/10.1021/acs.jafc.8b00692

Liu, M., Tang, Y., Zhao, K., Liu, Y., Guo, X., Ren, D., Yao, W., Tian, X., Gu, Y., Yi, B., \& Zhang, X. 2017. Determination of the fungal community of pit mud in fermentation cellars for Chinese strong-flavor liquor, using DGGE and Illumina MiSeq sequencing. Food Research International, 91, 80-87. https://doi.org/10.1016/j.foodres.2016.11.037

Liu, M. K., Tang, Y. M., Guo, X. J., Zhao, K., Tian, X. H., Liu, Y., Yao, W. C., Deng, B., Ren, D. Q., \& Zhang, X. P. 2017. Deep sequencing reveals high bacterial diversity and phylogenetic novelty in pit mud from Luzhou Laojiao cellars for Chinese strong-flavor Baijiu. Food Research International, 102, 68-76. https://doi.org/10.1016/j.foodres.2017.09.075

Liu, M. K., Tang, Y. M., Zhao, K., Gu Y. F., Ren, D. Q., Yao, W. C., Tian, X. H., Liu, Y., \& Zhang, X. P. 2017. Recent advances in research on the community, isolation, and application of microbes in the pit mud used in manufacture of Chinese Strong-flavor Baijiu. Microbiology China, 44 (5), 1222-1229. https://doi.org/10.13344/j.microbiol.china.160559

Magoč, T., \& Salzberg, S. L. 2011. FLASH: fast length adjustment of short reads to improve genome assemblies. Bioinformatics, 27(21), 2957-2963. https://doi.org/10.1093/bioinformatics/btr507

McGovern, P. E., Zhang, J., Tang, J., Zhang, Z., Hall, G. R., Moreau, R. A., Nunez, A., Butrym, E. D., Richards, M. P., Wang, C. S., Cheng, G., Zhao, Z., \& Wang, C. 2004. Fermented beverages of pre- and proto-historic China. Proceedings of the National Academy of Sciences of the United States of America, 101 (51), 17593-17598. https://doi.org/10.1073/pnas.0407921102

Oksanen, J., Blanchet, F. G., Kindt, R., Legendre, P., Minchin, P. R., O'Hara, R. B., Simpson, G. L., Solymos, P., Stevens, M. H. H., Wagner, H. H. 2018. Package. Vegan: community ecology package. https://cran. r-project. org/web/packages/vegan/index. Html

Plugge, C. M., Balk, M., Zoetendal, E. G., \& Stams, A. J. 2002. Gelria glutamica gen. nov., sp. nov., a thermophilic, obligately syntrophic, glutamate-degrading anaerobe. International journal of systematic and evolutionary microbiology, 52(2), 401-407. https://doi.org/10.1099/00207713-52-2-401

Quast, C., Pruesse, E., Yilmaz, P., Gerken, J., Schweer, T., Yarza, P., Peplies, J., \& Glöckner, F. O. 2012. The SILVA ribosomal RNA gene database project: improved data processing and 
452

453

454

455

456

457

458

459

460

461

462

463

464

465

466

467

468

469

470

471

472

473

474

475

476

477

478

479

480

481

482

483

484

485

486

487

488

489

490

491

web-based tools. Nucleic acids research, 41(D1), D590-D596.

https://doi.org/10.1093/nar/gks 1219

Segata, N., Izard, J., Waldron, L., Gevers, D., Miropolsky, L., Garrett, W. S., \& Huttenhower, C. 2011. Metagenomic biomarker discovery and explanation. Genome Biology, 12 (6), R60. https://doi.org/10.1186/gb-2011-12-6-r60

Shannon, C. E. 1997. The mathematical theory of communication. 1963. MD Computers, 14 (4), 306-317. https://doi.org/10.1063/1.3067010

Shen, Y. F. 2014. Handbook of Chinese liquor making technology (2nd ed). Beijing: China Light Industry Press.

Tang S. Y., Wang R., Chen X. W., Long P., Wang F. \& Chen L. 2012. Review of Wuliangye old pit mud Research. Food and Fermentation Technology, 48 (6): 7-11. https://doi.org/10.3969/j.issn.1674-506X.2012.06-00

Tao, Y., Li, J. B., Rui, J. P., Xu, Z. C., Zhou, Y., Hu, X. H., Wang, X., Liu, M. H., Li, D. P., \& Li, X. Z. 2014. Prokaryotic Communities in Pit Mud from Different-Aged Cellars Used for the Production of Chinese Strong-Flavored Liquor. Applied and environmental microbiology, 80 (7), 2254-2260. https://doi.org/10.1128/AEM.04070-13

Tao, Y., Rui, J. P., Li, J. B., Xu, Z. C., Li, D. P., Tang, Q. L., Wei, Y., \& Li, X. Z. 2014. Microbial community compositions and diversity in pit mud of Chinese Luzhou-flavor liquor. CIESC Journal, 65 (5), 1801-1807. https://doi.org/10.3969/j.issn.04381157.2014.05.034

Wang, L., Wang, Y. Y., Wang, H. Y., Liu, G., Yang, F., Jiang, H. G., Jin, X. U., Wang, D. Q., \& Jin, T. 2015. Microbial Composition of Bottom Pit Mud in Jiangxiang Baijiu(Liquor) Pit. Liquor Making Science \& Technology (1), 12-15. https://doi.org/10.13746/j.njkj.2014513

Wang, Q., Zhang, H., \& Liu, X. 2016. Microbial Community Composition Associated with Maotai Liquor Fermentation. Journal of Food Science, 81 (6), M1485-1494. https://doi.org/10.1111/1750-3841.13319

Wang, X., Du, H., \& Xu, Y. 2017. Source tracking of prokaryotic communities in fermented grain of Chinese strong-flavor liquor. International Journal of Food Microbiology, 244, 27 35. https://doi.org/10.1016/j.ijfoodmicro.2016.12.018

Wu, Y. Y., Qi, Y. P., Xue, C. J., Guo, S. Z., \& Jie, L. X. 1980. Microbial ecology distribution and anaerobic fermentation characteristics in the pit mud of Luzhou-flavor liquor. Microbiology China (3), 108-112. https://doi.org/10.13344/j.microbiol.china.2007.02.013

Yang, M. M., Chen, L., Liu, Q., Li, H., Hu, Y. J., Liu, Y. M., \& Yang, Z. J. 2017. Correlation analysis between physicochemical indexes and sensory evaluation of the geographical indication product "Yibin Jiu" base liquor. China Brewing, 36 (01), 142-145. https://doi.org/10.11882/j.issn.0254-5071.2017.01.030

Ye, G. B., Luo, H. B., Yang, X. D., Li, D. Y., Wang, Y., \& Ni, B. 2013. Community Stricture of Prokaryotes in Pit Mud of Lu-Flavor Liquor from Luzhou Prefecture Based on Cultureindependent Approach. Journal of Food Science, 34 (17). https://doi.org/10.7506/spkx10026630-201317038 
492

493

494

495

496

497

498

499

500

501

502

503

504

505

506

507

508

509

510

511

512

513

514

515

516

517

518

519

520

521

522

523

524

You, L., Wang, S., Zhou, R., Hu, X., Chu, Y., \& Wang, T. 2016. Characteristics of yeast flora in Chinese strong-flavoured liquor fermentation in the Yibin region of China. Journal of the Institute of Brewing, 122 (3), 517-523. https://doi.org/10.1002/jib.352

Yue, Y. Y., Hang, W. X., Liu, X., Hu, C., \& Zheng, S. Y. 2007. Isolation and Identification of Facultative Anaerobes in the Pit Mud of Chinese Luzhou-flavor Liquor. Microbiology China, 34 (2), 251-255. https://doi.org/10.3969/j.issn.0253-2654.2007.02.013

Zhao, D., Zheng, J., Peng, Z. Y., Lv, X. L., Yang, K. Z. \& Zhang, J. M. 2017. Prokaryotic microbial community in wuliangye pit mud using nextseq high-throughput sequencing technology. Food and Fermentation Industries, (09), 5-12. https://doi.org/10.13995/j.cnki.11-1802/ts.014156

Zhao, J. S., Zheng, J., Zhou, R. Q., \& Shi, B. 2012. Microbial community structure of pit mud in a Chinese strong aromatic liquor fermentation pit. Journal of the Institute of Brewing, 118 (4), 356-360. https://doi.org/10.1002/jib.52

Zhao X. L., Zhou Y. K., Qiao Z. W., Li H., Li W. H., Liu B. H., Yang Q. S., Zhang L. J., Du S. T., Xu Z. H. \& Yang J. Z. 2013. A Study on Unique Climate Characteristics of Yibin of Sichuan in Liquor Production. Liquor Making, (5):5-10.

Zheng, J., Liang, R., Zhang, L., Wu, C., Zhou, R., \& Liao, X. 2013. Characterization of microbial communities in strong aromatic liquor fermentation pit muds of different ages assessed by combined DGGE and PLFA analyses. Food Research International, 54 (1), 660-666. https://doi.org/10.1016/j.foodres.2013.07.058

Zheng, Q., Lin, B., Wang, Y., Zhang, Q., He, X., Yang, P., Zhou, J., Guan, X., \& Huang, X. 2015. Proteomic and high-throughput analysis of protein expression and microbial diversity of microbes from 30- and 300-year pit muds of Chinese Luzhou-flavor liquor. Food Research International, 75, 305-314. https://doi.org/10.1016/j.foodres.2015.06.029

Zheng, X. W., \& Han, B. Z. 2016. Baijiu (白酒), Chinese liquor: History, classification and manufacture. Journal of Ethnic Foods, 3 (1), 19-25. https://doi.org/10.1016/j.jef.2016.03.001

Zhu L. L., Luo H. B., Huang Z. G., Wei, C. H., Deng J., Xie J. 2018. Screening of physicochemical indicators for pit mud quality evaluation in strong-flavor Baijiu. China Brewing, 37(12), 33-39. https://doi.org/10.11882/j.issn.0254-5071.2018.12.007

Zou, W., Zhao, C., \& Luo, H. 2018. Diversity and Function of Microbial Community in Chinese Strong-Flavor Baijiu Ecosystem: A Review. Front Microbiol, 9, 671. https://doi.org/10.3389/fmicb.2018.00671 


\section{Table 1 (on next page)}

Number of classified species at different taxa 
1

Table 1 Number of classified species at different taxa

\begin{tabular}{llllll}
\hline \multicolumn{1}{c}{ Group } & Phylum & Class & Order & Family & Genus \\
\hline Middle & 45 & 88 & 151 & 291 & 680 \\
Sub & 46 & 88 & 160 & 304 & 713 \\
All & 49 & 94 & 172 & 332 & 811 \\
\hline
\end{tabular}

2 


\section{Table 2 (on next page)}

Comparison of dominant microorganism in PM in different regions of strong-aroma Baijiu

*Ratio: The ratio of the number of dominant genera which shared with "Yibin Baijiu" pit mud to the total number of dominant genera. 
1 Table 2 Comparison of dominant microorganism in PM in different regions of strong-aroma Baijiu

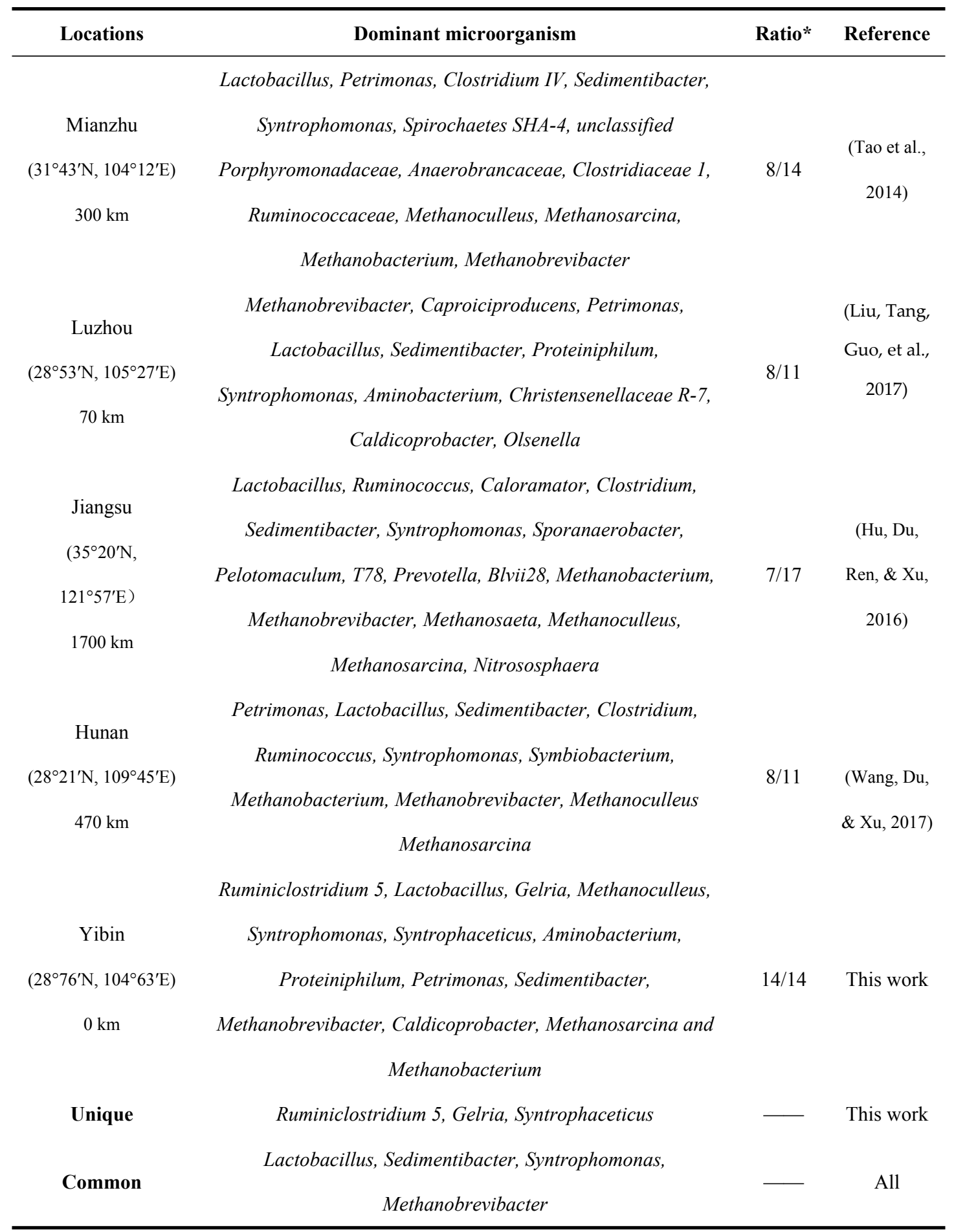

2 *Ratio: The ratio of the number of dominant genera which shared with "Yibin Baijiu" pit mud to the total 3 number of dominant genera. 
Table 3(on next page)

Comparison of dominant microorganism in PM of different aroma types of Baijiu 
Table 3 Comparison of dominant microorganism in PM of different aroma types of Baijiu

\begin{tabular}{|c|c|c|}
\hline Representative aroma & Dominant microorganism & Reference \\
\hline miscellaneous-aroma & $\begin{array}{l}\text { Corynebacterium, Myroides, Sphingobacterium, Lactobacillus, } \\
\text { Clostridium, Acetobacter, Alcaligenes, Enterobacter and } \\
\text { Acinetobacter }\end{array}$ & (Huang et al., 2017) \\
\hline sauce-aroma & $\begin{array}{l}\text { Clostridiaceae, Ruminococcaceae and Thermoanaerobacteriaceae } \\
\text { Methanoculleus, Methanosarcina, Methanosaeta and } \\
\qquad \text { Methanobacterium } \\
\text { Weissella, Lactobacillus, Bacillus, Enterobacter and Paenibacillus }\end{array}$ & $\begin{array}{l}\text { (Wang et al., 2015) } \\
\text { (Bian, Ye, Yang, Ni, \& } \\
\text { Li, 2012) } \\
\text { (Wang, Zhang, \& Liu, } \\
\text { 2016) }\end{array}$ \\
\hline $\begin{array}{l}\text { strong-aroma } \\
\text { (common) }\end{array}$ & $\begin{array}{c}\text { Lactobacillus, Sedimentibacter, Syntrophomonas and } \\
\text { Methanobrevibacter }\end{array}$ & - \\
\hline
\end{tabular}

2 
Figure 1

Chaol richness indices (a) and Shannon diversity indices (b).

The box-plots exhibit the first (25\%) and third (75\%) quartiles, the median, and the maximum and minimum observed values within each data set.
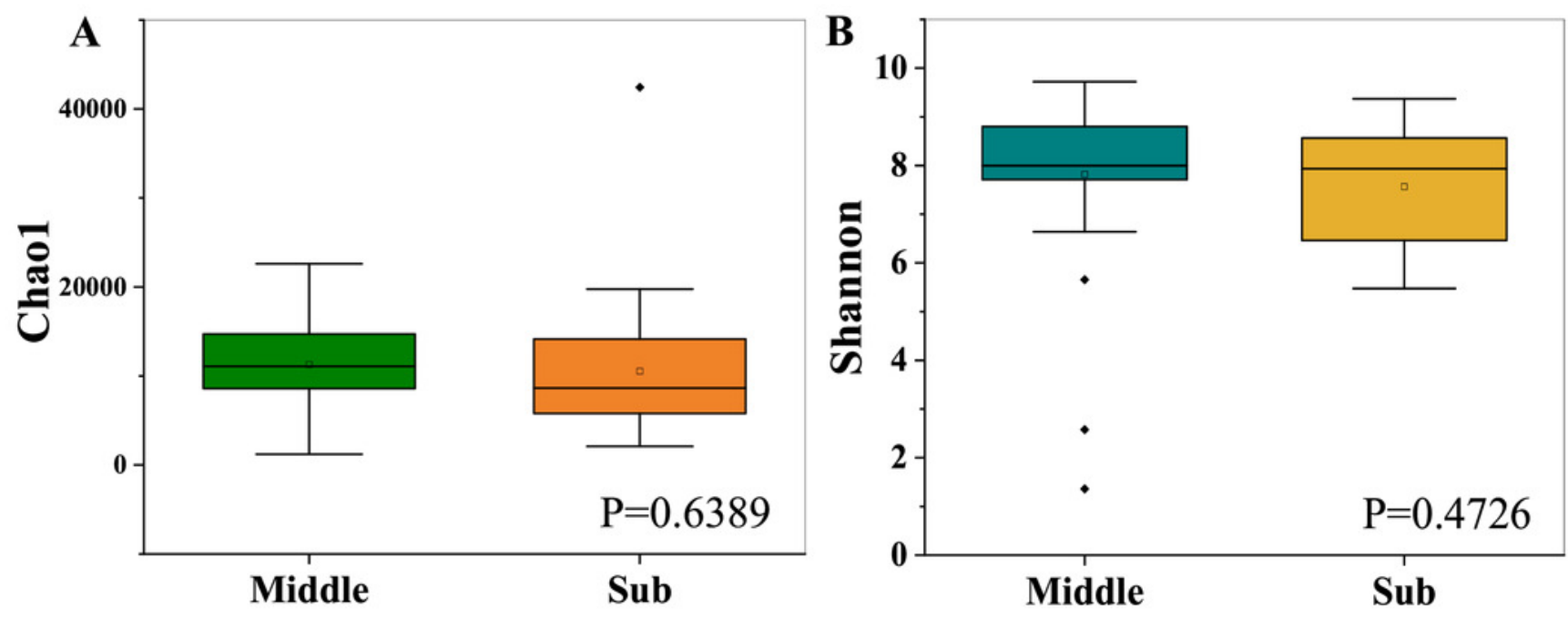
Figure 2

PCoA of weighted Unifrac distance (a) and unweighted Unifrac distance (b) for the microbiota at PM of middle-layer group and sub-layer group.

The principal coordinate combination with the highest contribution rate was selected for display.
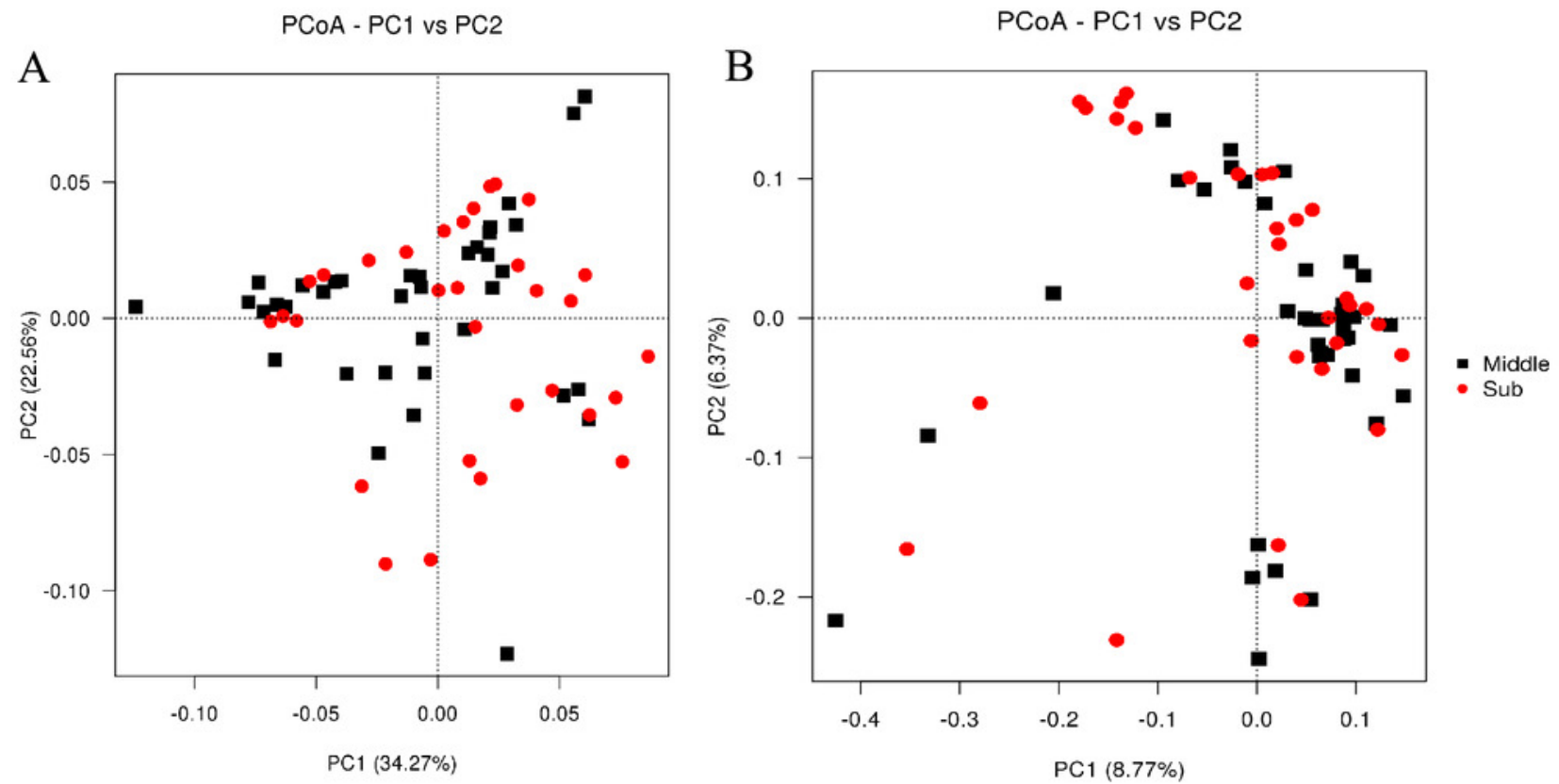
Figure 3

Taxonomic classifications of the bacterial communities in PM samples collected from sub-layer and middle-layer at the phylum level.

The "Others" refer to the group that could not be accurately assigned to any known bacterial taxonomic group at the phylum level, as well as the group that ranked after 10 phyla with most relative abundance.

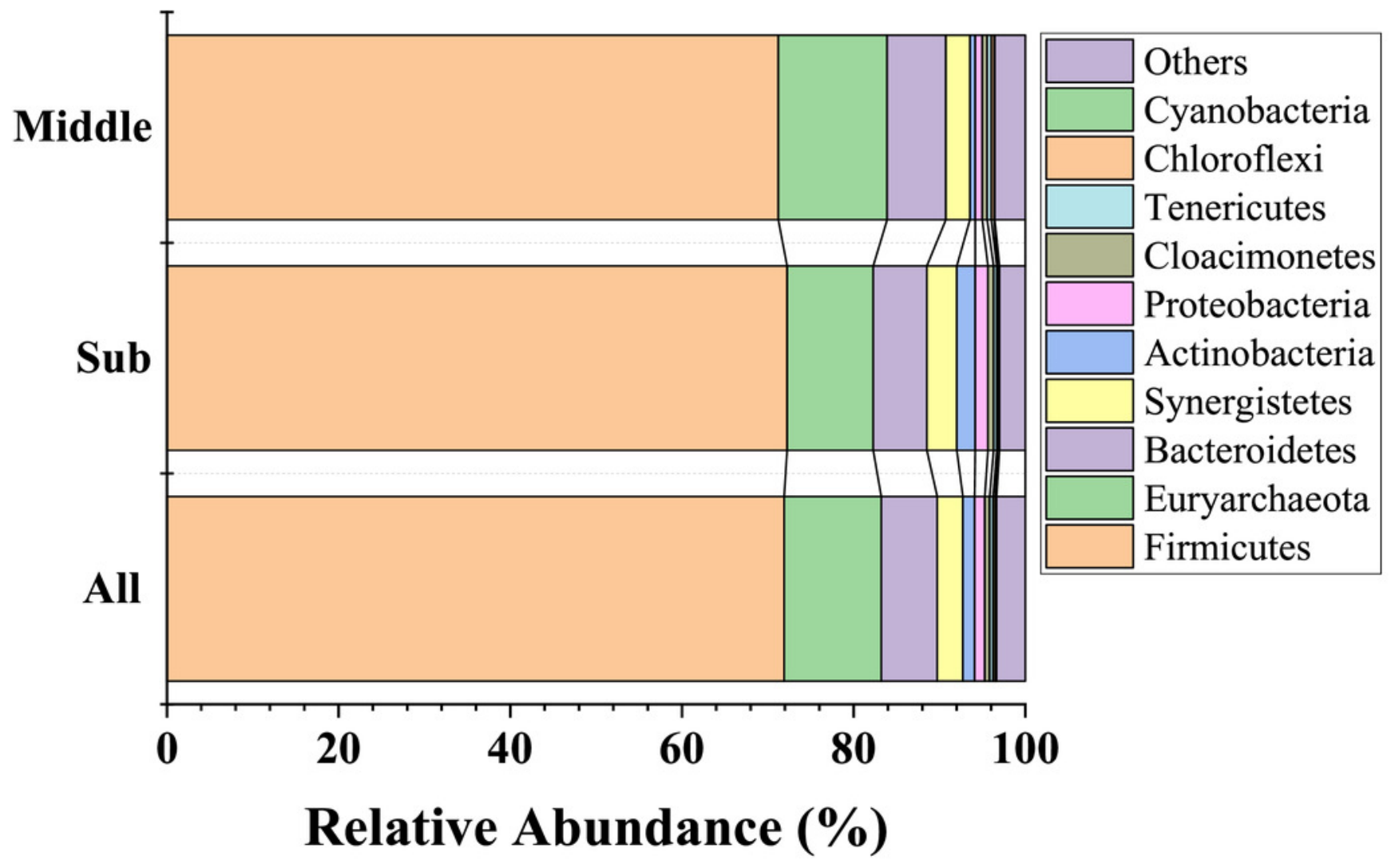




\section{Figure 4}

The ten most abundant genera in bacterial community at the genus level.

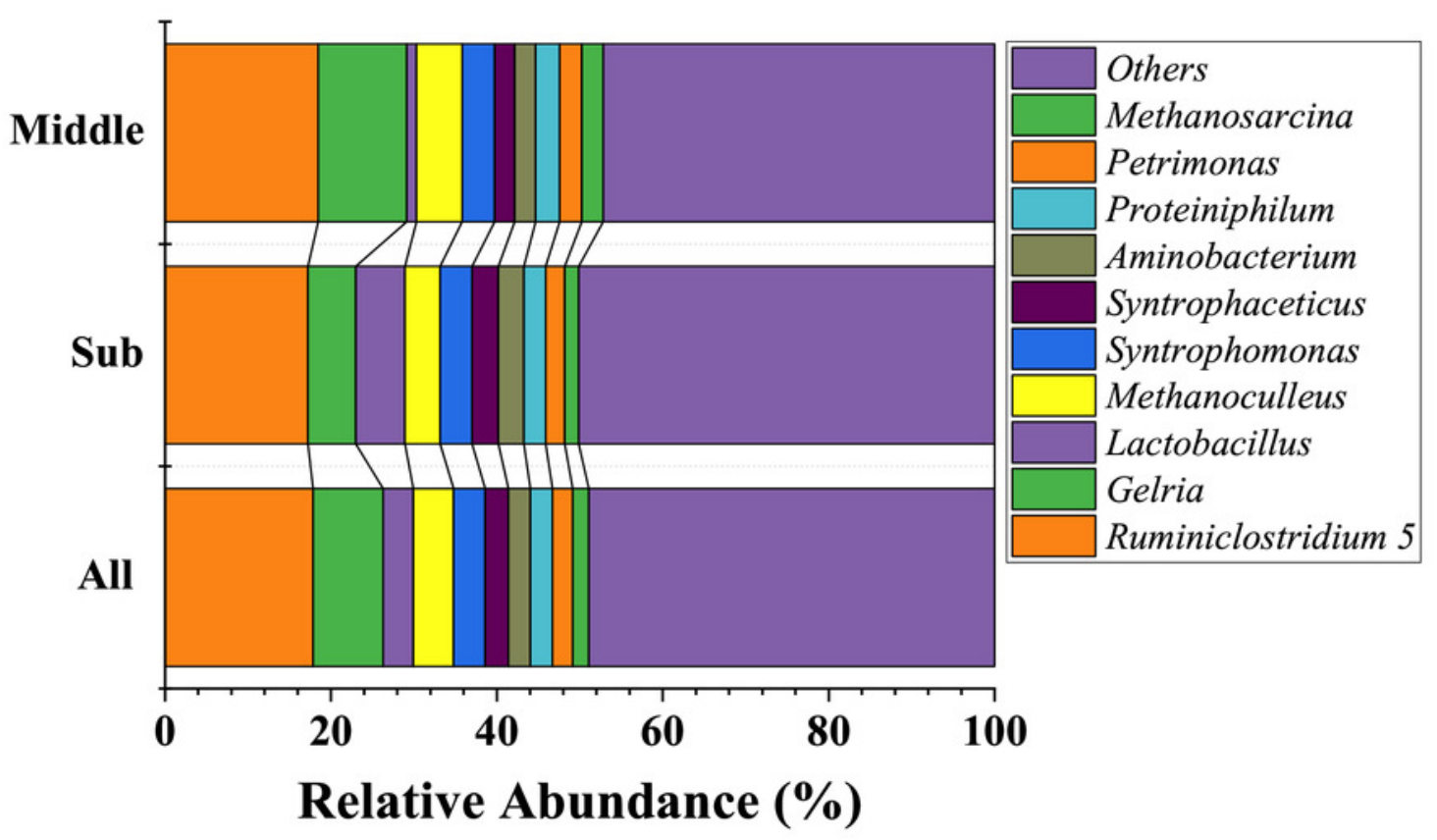




\section{Figure 5}

LDA score map with a threshold value of 4.0.

The length of the histogram represents the effect size of the species with significant difference in abundance in different groups.

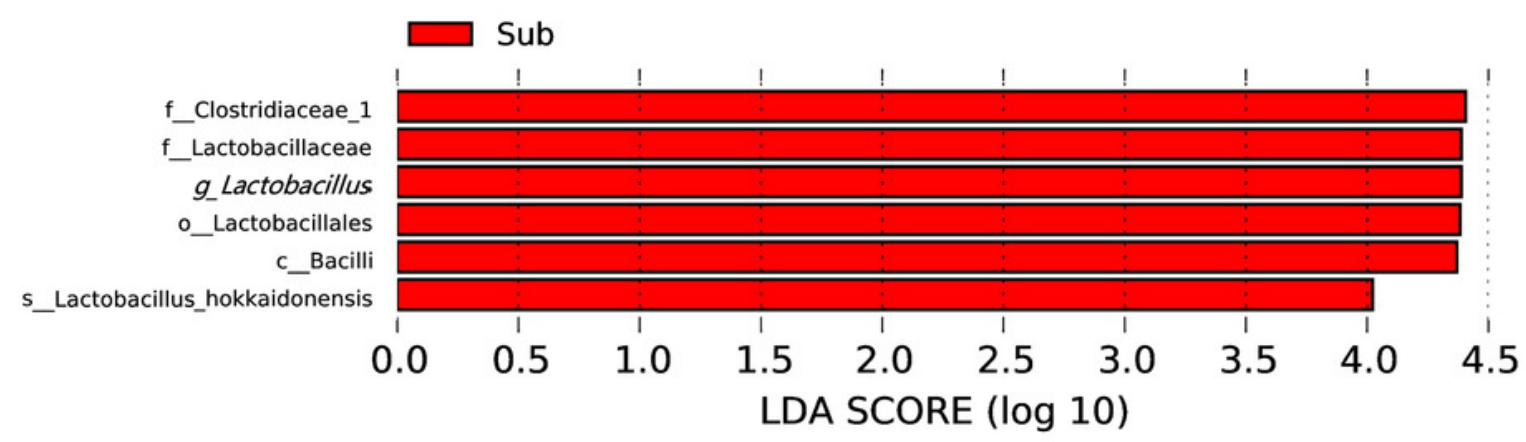




\section{Figure 6}

Heat map showing the Spearman's correlation coefficient of environmental factors and bacterial community in PM.

The horizontal direction shows the species information, the longitudinal direction shows the environmental factor information, the heat map corresponds to the Spearman's correlation coefficient $R$, and the $R$ value ranges from -1 to 1 . $R<0$ indicated negative correlation and $R$ $>0$ indicated positive correlation. 


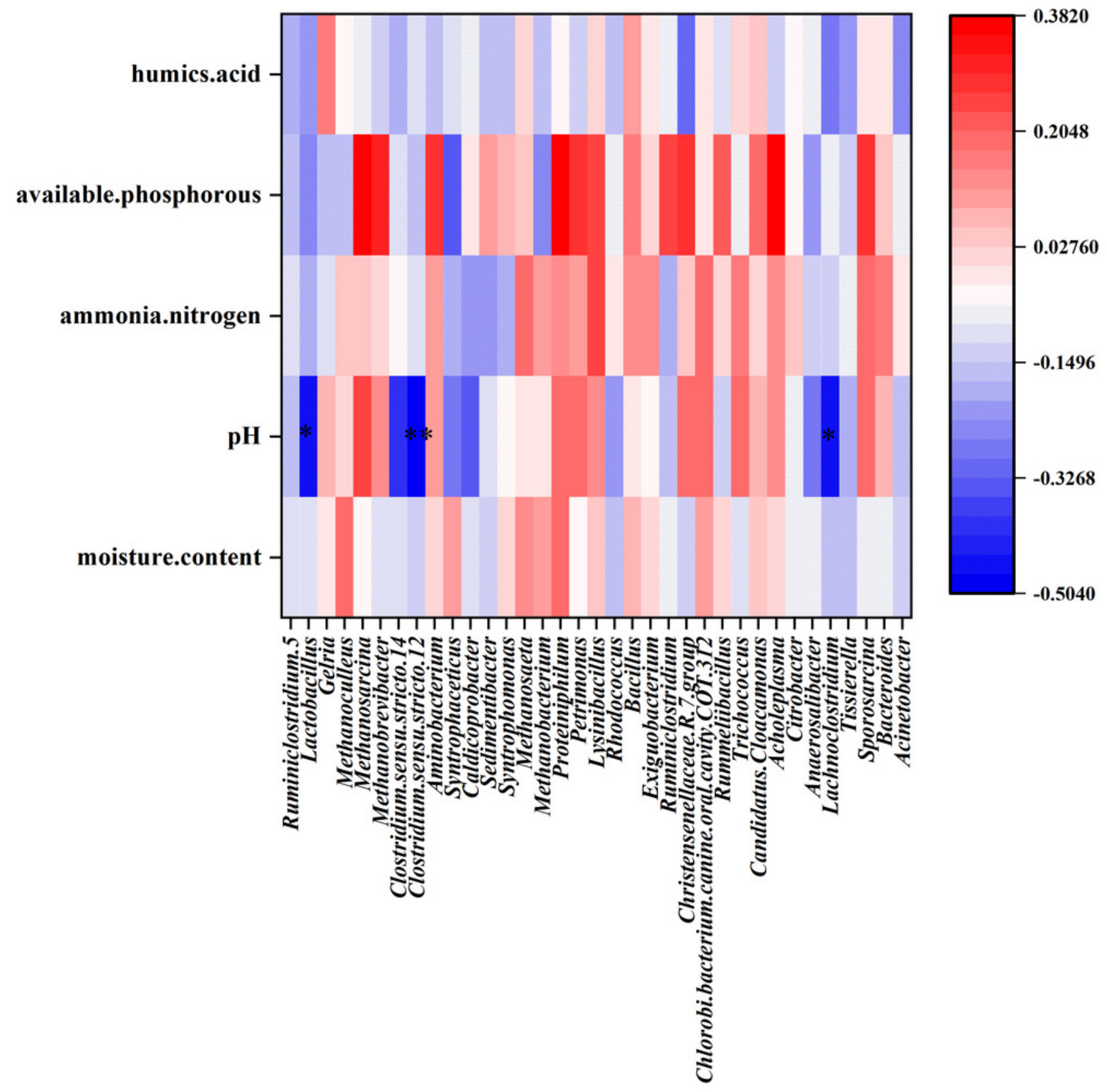

